\title{
OTIMIZAÇÃO DE PERFORMANCE NO SISTEMA DE LAVAGEM DE GAS DO ALTO FORNO*
}

\author{
Daniel Rezende Machado ${ }^{1}$ \\ Osvaldo Benedito Dib Melo² \\ Adilio Freitas dos Santos ${ }^{3}$ \\ Walter Bruno Oliveira ${ }^{4}$ \\ Saulo Teles Peixoto ${ }^{5}$ \\ Augusto Luis Marucci ${ }^{6}$ \\ Alexandre Magno Barbosa Moreira ${ }^{7}$
}

\section{Resumo}

O trabalho tem por objetivo de mostrar uma otimização da performance de sistema de lavagem de gases (conjunto espessador e filtro a vácuo) do Alto Forno de uma siderúrgica localizada no Nordeste, a CSP, Cia Siderúrgica do Pecem. A unidade entrou em operação no ano de 2016, inicialmente com orientações/informações de projeto, tendo muito desvios no controle da turbidez e sólidos suspensos da água da saída do espessador, em função da tecnologia aplicada, que não estava funcionando adequadamente para a realidade da região e ocasionava custo elevado, além de muitos problemas operacionais. A metodologia aplicada baseou-se em teste na planta com produtos químicos, ensaio de laboratório com teste de jarro e análises físico-químicas da água de recirculação. Os resultados obtidos foram excelentes na redução da turbidez e sólidos suspensos, aumento da retirada de lama pelo filtro rotativo, maior tempo de uso da manta dos filtros a vácuo e uma redução de custo com produto químicos e mão de obra, totalizando uma economia significativa em reais/ano.

Palavras-chave: Alto forno ; Sistema de lavagem de gás; Espessadores ; Filtro a vácuo.

\section{PERFORMANCE OTIMIZATION IN BLAST FURNANCE GAS CLEANING SYSTEM Abstract}

The following work has the objective of showing a performance optimization in a gas cleaning process (thickeners and vacuum filter) in the blast furnace of a steel plant located in the Northeast, CSP, Cia Siderúrgica do Pecem. The unit came on stream in the year 2016 with project guidelines / information and had many divergences to be overcome as the initial application technology did not work properly for the region's reality and caused high cost and many operational problems. The methodology was test in the plant with chemical products, jug test and physical-chemical analyzes of the recirculation water. The results were excellent with a reduction of cost with chemical products, blanket in vacuum filters and labor, totaling a very important saving/year.

Keywords: Blast Furnance; Gas Cleaner Process, Tickener, Vacuum Filter.

1 Engenheiro Metalúrgico, coordenador de equipamento, Alto Forno da Cia Siderúrgica do Pecem, CSP, São Gonçalo do Amarante, Ceará, Brasil.

2 Engenheiro Químico, Especialista, Gerente de Contas, GE Water \& Process Technologies, Fortaleza, Ceará, Brasil.

3 Tecnólogo em Processo Gerenciais, Técnico de Operação 3, Alto Forno da Cia Siderúrgica do Pecem, CSP, São Gonçalo do Amarante, Ceará, Brasil .

4 Técnico em Eletromecânica, Técnico de Produção, Alto Forno da Cia Siderúrgica do Pecem, CSP, São Gonçalo do Amarante, Ceará, Brasil.

5 Engenheiro Mecânico, Analista de equipamento pleno, Alto Forno da Siderúrgica do Pecem, CSP, São Gonçalo do Amarante, Ceará, Brasil.

6 Engenheiro Mecânico, Consultor de Siderurgia para Latina América, GE Water \& Process Technologies, São Paulo, SP, Brasil.

7 Engenheiro Químico, Gerente de aplicação WWT e MemChem Latin America, GE Water \& Process Technologies, São Paulo, SP, Brasil. 


\section{INTRODUÇÃO}

O sistema de lavagem de gás do alto forno (GCP) da CSP tem dois espessadores TH 951 e 952, com volume de $3300 \mathrm{~m}^{3}$ e uma vazão de recirculação máxima de 1200 $\mathrm{m}^{3} / \mathrm{h}^{1}$. A temperatura da água que pode chegar até $70^{\circ} \mathrm{C}^{2}$ e opera com tratamento químico para evitar entupimento dos doze bicos aspersores. Nos espessadores a água de retorno do processo, junto com a dosagem de polímero e produto químico, retira os sólidos que vem do gás do alto forno.

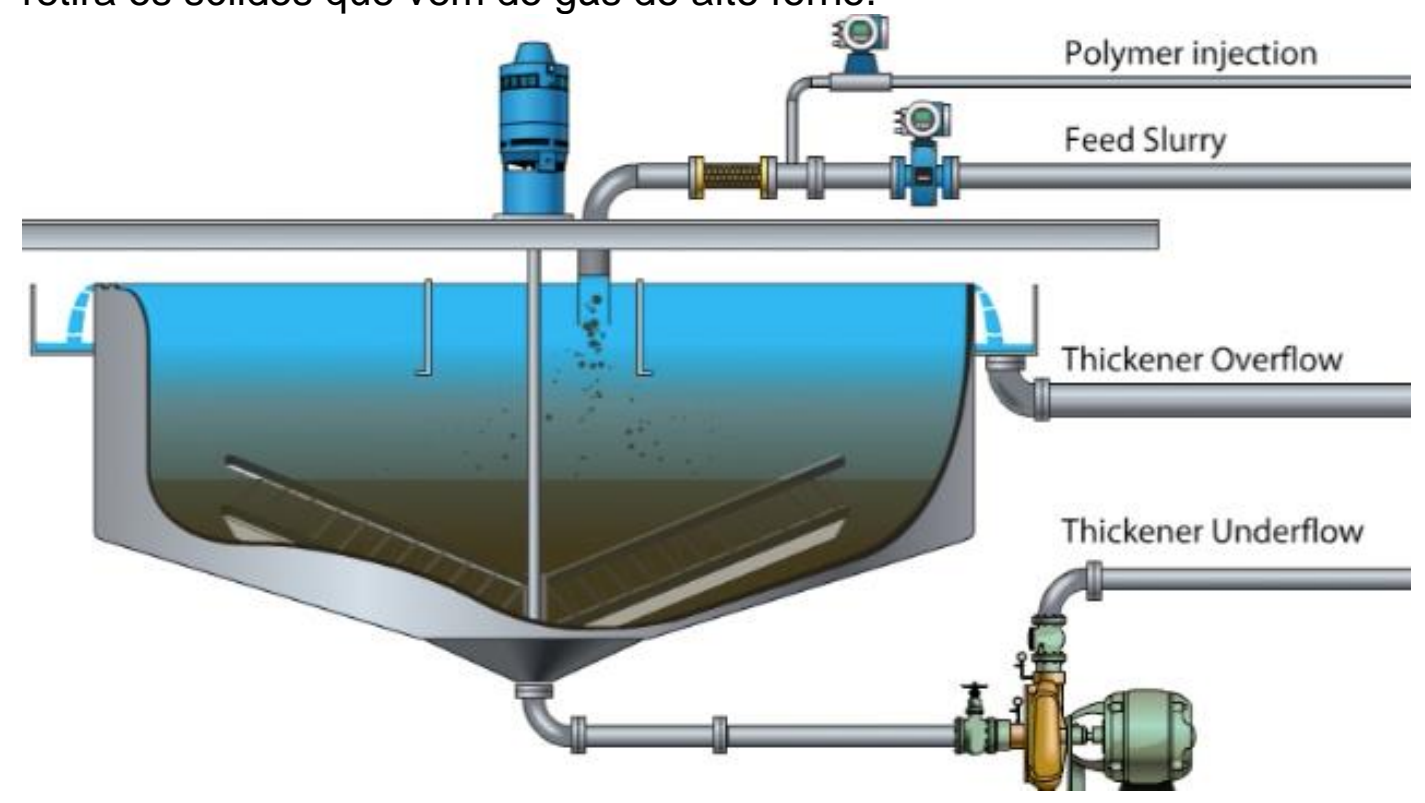

Figura 1 : llustração de partes importantes do espessador ${ }^{3}$.

A função principal do espessador é reduzir a velocidade da água recirculante, permitindo um fluxo lamelar, provocando a sedimentação dos sólidos e ocasionando a compactação da lama no fundo, logo, reduzindo a possibilidade de entupimento dos bicos aspersores do lavador de gás. A lama do fundo do espessador é enviada para um filtro a vácuo. A temperatura elevada da água de recirculação do espessador sofre evaporação parcial, aumentando a concentração de sais e de subprodutos do topo do alto forno, necessitando descarga para desconcentrar a água de recirculação do GCP e facilitar a sua performance.

A operação inicial apresentou problemas de performance e custo elevado de produtos químicos para tratamento da água de recirculação. As informações e orientações técnica da partida do GCP não estava atingindo os resultados esperados no processo de clarificação da água de recirculação do lavador de gás. $O$ trabalho mostra a evolução e os resultados da otimização das condições operacionais realizada ao longo do ano de 2016 no GCP da CSP.

A condição operacional inicial era dosagem de soda caústica para correção de $\mathrm{pH}$, aplicação de polimero para remoção de lama, controle de temperarura da água no máximo de $40^{\circ} \mathrm{C}$ e condutividade operacional abaixo de 2500 microsiemens. Não havia previsão de coagulante para auxiliar na clarificação. A tabela 01 mostra os valores indicado para a operação da água de recirculação do GCP. 
Tabela 01 : Parametros de controle recomendados inicialmente ${ }^{4}$.

\begin{tabular}{|c|c|c|c|}
\hline Parametror & Poramelens & Unid & $\begin{array}{l}\text { GCP } \\
\text { water }\end{array}$ \\
\hline $\mathrm{pH}$ & $\mathrm{pH}$ & & $6.0-8.0$ \\
\hline Temperatura & Temperature & ${ }^{\circ C}$ & $45 !$ \\
\hline Cor & Color & Degree & - \\
\hline Turbidez & Turbity & NTU & - \\
\hline Dureza & Ca-Hardness & $\mathrm{ppm}$ & - \\
\hline Oleo & 예 & $\mathrm{mg} / \mathrm{h}$ & - \\
\hline $\mathrm{SO}_{4}$ & 504 & $\mathrm{mg} / \mathrm{L}$ & - \\
\hline Ferro & $\mathrm{fe}$ & ppm & $40 !$ \\
\hline Soldos Totais & Total Solds & $\mathrm{mg} / \mathrm{h}$ & - \\
\hline ss & $\begin{array}{l}\text { Suspended } \\
\text { Solids }\end{array}$ & $m g / t$ & - \\
\hline $\begin{array}{l}\text { Consumo de } \\
\text { KMnOA }\end{array}$ & $\begin{array}{c}\text { Consuption of } \\
\text { KMnO4 }\end{array}$ & $\mathrm{mg} / \mathrm{L}$ & - \\
\hline 5102 & 5102 & $\mathrm{mg} / \mathrm{h}$ & - \\
\hline Amónio & $\mathrm{NH} 4$ & $\mathrm{mg} / \mathrm{L}$ & . \\
\hline Cl. & Cl. & $\mathrm{ppm}$ & - \\
\hline Pressōo Máximo & Maxpressure & $\mathrm{Ka} / \mathrm{cm} 2$ & 10.0 \\
\hline Pressoo Minima & Min pressure & $\mathrm{Ka} / \mathrm{cm} 2$ & - \\
\hline Condut. Eletrica & $\begin{array}{l}\text { Eletric } \\
\text { Conductivity }\end{array}$ & $\mathrm{ms} / \mathrm{m}$ & 2001 \\
\hline
\end{tabular}

Nos filtros rotativos não foi recomendado nenhuma dosagem de produto quimico, apenas remoção das mantas quando rompida/furadas. Espessura da lama conseguida inicialmente era inferior a $2 \mathrm{~mm}$, o que não permitia uma remoção adequada da lama no raspador do filtro. As condições operacionais eram de pressão de vacuo variando de $-0,8$ a $-0,6 \mathrm{MP}$,pressão de purga igual 2,0 MP, rotação do filtro de 30 a $50 \mathrm{rpm}$, conforme densidade da lama. Opera 24 horas, com 3 horas em cada uma das quatro bombas. A baixa remoção de lama acarreta um aumento de carga no eixo do agitador do espessador que ocasiona aumento da amperagem. Obrigando a parada do equipamento, conforme figura 02, para que seja feito uma limpeza do espessador. A operação de limpeza requer equipamentos pesados e especificos para remoção da lama no fundo do espessador. O processo de limpeza tem custo elevado e tempo longo (2 a 3 dias), oferecendo risco de sobrecarga no outro espessador em operação.

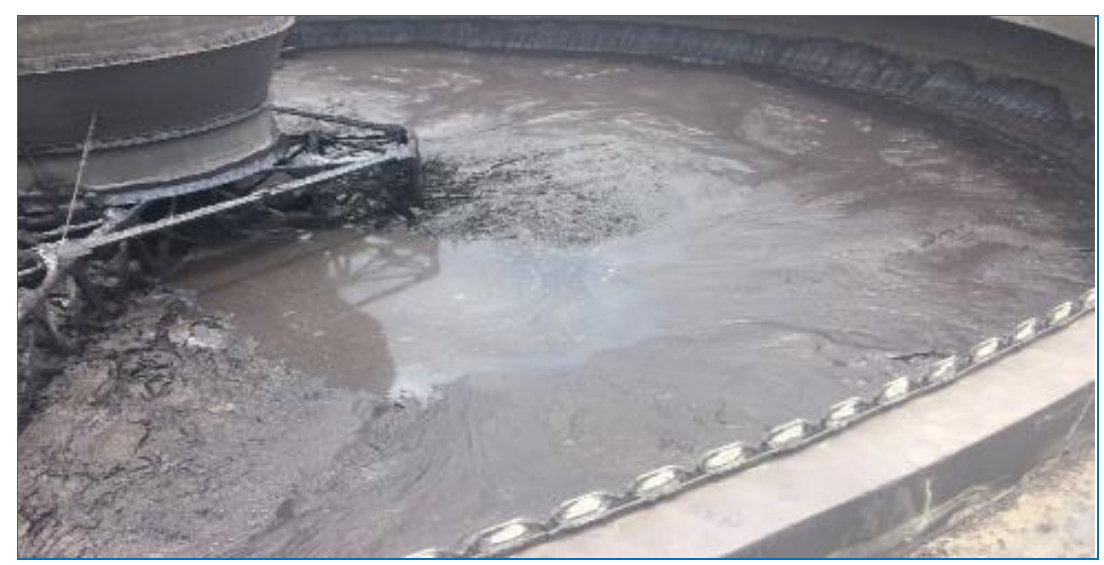

Figura 02 : Vista do espessador sem água, durante a parada para $1^{\text {a }}$ lavagem em agosto/2016.

$\mathrm{Na}$ partida da planta foram alteradas as dosagens de produtos pela CSP para conseguir os resultados de turbidez e sólidos suspenso recomendado como ideal na 
saída do espessador, respectivamente, abaixo de 100 NTU e 100 ppm. Optamos por usar a turbidez no controle de qualidade do processo de clarificação pois a metodologia de análise da turbidez ser mais rápida em relação ao de sólidos suspensos (SS) para avaliação de campo. A proposta inicial não foi mantida pois os resultados da saída da água estavam com valores de turbidez e SS muito alto, o que iria gerar um problema de erosão para os bicos e perda de performance da lavagem dos gases.

A tabela 02 mostra alguns resultados da água de saída dos espessadores com o tratamento do projeto com elevado de teor de turbidez e de condutividade. Encontramos resultados de turbidez e SS dos espessadores com problemas de clarificação, atingindo valores de turbidez acima de 1320 ppm NTU e 1000 ppm de SS no mês de agosto de 2016.

Tabela 02:Alguns valores de condutividade, $\mathrm{pH}$ e turbidez encontrado durante a operação de partida ${ }^{5}$.

$\begin{array}{cccc} & \text { Cond } & \text { ph } & \text { turb } \\ \text { Espessador A } & 25.74 \mathrm{~ms} / \mathrm{cm} & 8,58 & 24 A \\ \text { Espessador B } & 25,35 \mathrm{~ms} / \mathrm{cm} & 8,49 & 703\end{array}$

\section{MATERIAIS E MÉTODOS}

Os equipamentos utilizados foram teste de jarro, teste de Resíduo Sedimentáveis, termômetro infravermelho, equipamento Hach 8900 para realização de análises físico-químicas no laboratório da GE Water na CSP e no laboratório central da CSP. O acompanhamento da planta e do processo foi realizado diariamente pela equipe da CSP e GE Water, para identificar parâmetros de controle, dosagem de produto e as modificações realizadas, gerando dados e informações para avaliação futuras.

\section{RESULTADOS E DISCUSSÃO}

Os resultados obtidos com os dados do projeto não foram satisfatórios e com elevado custo operacional, obrigando a CSP a fazer alterações no processo. $O$ aumento da condutividade da água de recirculação, conforme mostrado na figura 03 , não permitia descarga para o efluente, o que dificulta o processo de clarificação.

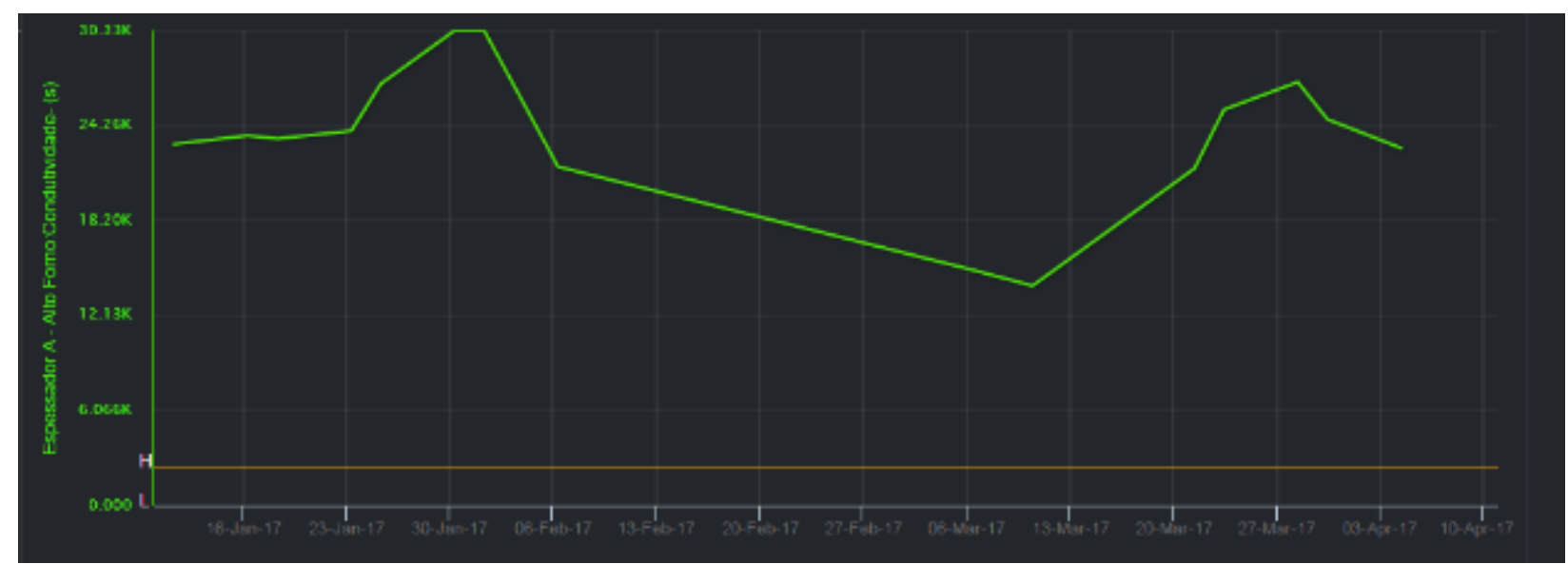

Figura 03 : Valores de condutividade acima de 12000 microsiemens do sistema durante 2016 e $2017^{6}$ 
Outro problema era que o tratamento inicial não resolvia o problema de qualidade da água no tocante a redução de concentração dos metais Chumbo $(\mathrm{Pb})$, Ferro $(\mathrm{Fe})$ e Zinco (Zn), o que impactava na não liberação de descarga da água para o efluente. Vários testes de jarro foram realizados na busca do melhor resultado para a planta. O teste com polímero sozinho não funcionou bem para a situação da água do espessador da CSP, conforme observado na figura 04. Os teste indicaram a necessidade da adição de policloreto de alumínio (PAC), junto com polímero da GE Water, conforme figura 05 , como a melhor opção para o GCP, aliado ao uso de soda para corrigir o $\mathrm{pH}$, quando necessário.

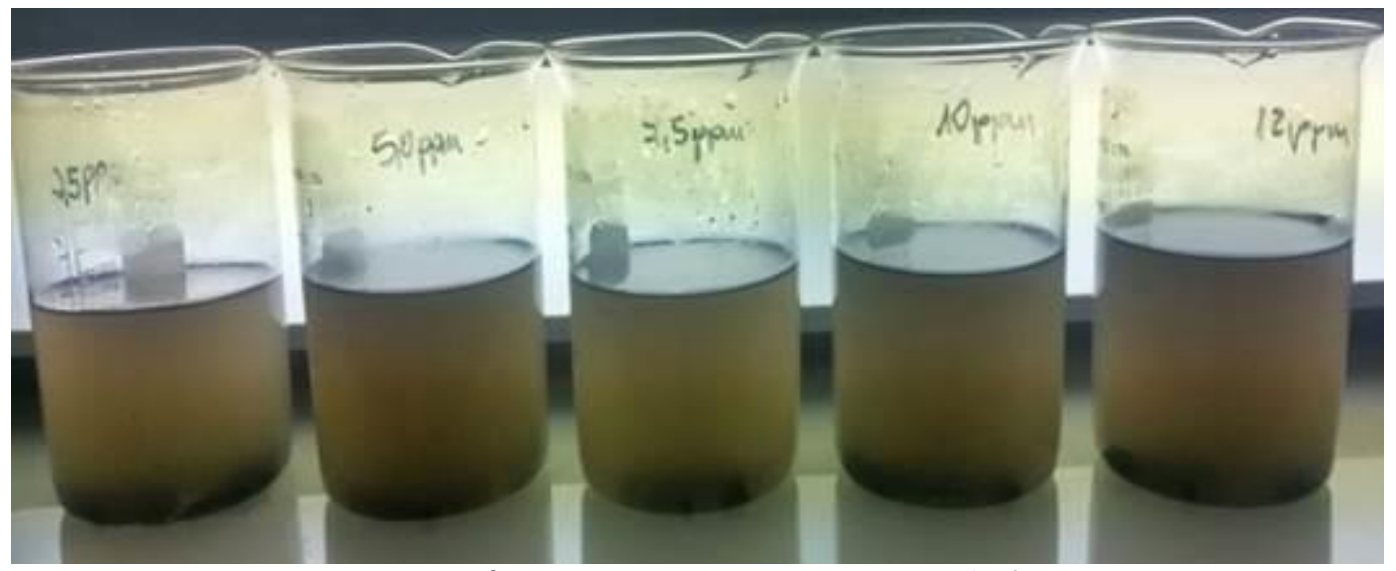

Figura 04:Teste com polímero sozinho indicando que não funcionando bem.

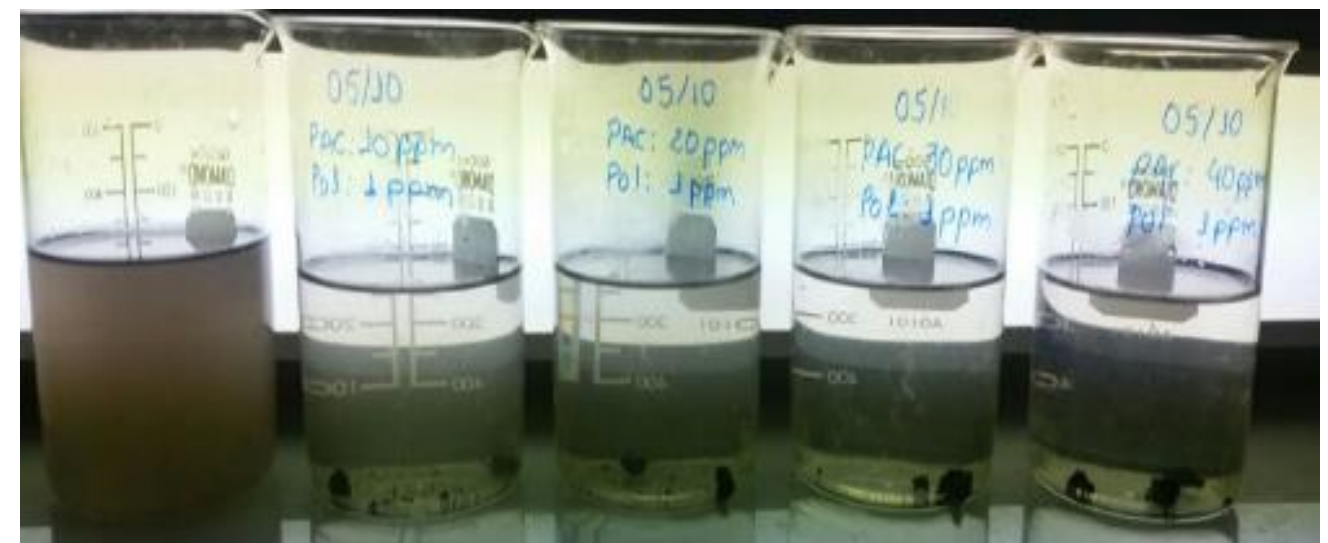

Figura 05:Teste com PAC com resultados de cor, SS e turbidez dentro da faixa recomendada.

A adição de PAC e controle de $\mathrm{pH}$ foram fundamentais para garantir o controle dos metais com valores abaixo do limite do processo, conforme mostra os valores de metais na tabela 03 , além de efluente com baixo sólidos suspensos e turbidez. Alterações nos pontos de dosagens foram também fundamental para otimização do processo e redução de custo. A dosagem do polímero estava indo preferencialmente para um único espessador, criando acumulo de lama no espessador "A " e obrigando a fazermos lavagem em intervalo de tempo curto, a cada 3 meses, ocasionando custo elevado para a unidade. 
Tabela 03 : Média de valores do início e os atuais na segunda linha da tabela ${ }^{5}$.

\begin{tabular}{|l|c|c|c|c|c|c|c|c|}
\hline Data da Coleta & $\mathrm{pH}$ & Condutividade & $\begin{array}{c}\text { Dureza Total } \\
(\mathrm{Ca}+\mathrm{Mg})\end{array}$ & $\begin{array}{c}\text { Fe Total } \\
(\mathrm{mg} / \mathrm{L})\end{array}$ & $\begin{array}{c}\text { PO4 } \\
(\mathrm{mg} / \mathrm{L})\end{array}$ & $\begin{array}{c}\text { SST } \\
(\mathrm{mg} / \mathrm{L})\end{array}$ & $\begin{array}{c}\mathrm{Pb} \\
(\mathrm{mg} / \mathrm{L})\end{array}$ & $\begin{array}{c}\mathrm{Zn} \\
(\mathrm{mg} / \mathrm{L})\end{array}$ \\
\hline $01 / 09 / 2016$ & 7,4 & $25.100,0$ & 858,6 & 93,1 & 12 & 2.250 & 32,3 & 647,3 \\
\hline $10 / 01 / 2017$ & $\mathbf{7 , 8}$ & $\mathbf{1 4 . 1 8 0 , 0}$ & $\mathbf{6 9 2}$ & $\mathbf{1 , 3}$ & $\mathbf{2 1}$ & $\mathbf{2 1 0}$ & $\mathbf{0 , 2 1}$ & $\mathbf{6 , 5}$ \\
\hline
\end{tabular}

A seguir a evolução de dosagens, algumas condições operacionais e custos dos produtos químicos no GCP explicado na tabela 04 . $\mathrm{Na}$ coluna atual é a recomendação da GE para o GCP. Na coluna projeto (previsto para operação inicial). Na coluna partida ( os primeiros 5 meses de operação).

Tabela 04: Comparativo de custo do tratamento do GCP da CSP.

\begin{tabular}{|l|c|c|c|c|}
\hline Produto & Unidade & Projeto & Partida & Atual \\
\hline Soda & $\mathrm{kg} / \mathrm{mês}$ & 1.200 & ${ }^{*} 0$ & 6.000 \\
\hline Polímero & $\mathrm{kg} / \mathrm{mês}$ & 30 & 3.200 & 504 \\
\hline Dispersante & $\mathrm{kg} / \mathrm{mês}$ & 700 & 4.500 & 1.260 \\
\hline PAC & $\mathrm{kg} / \mathrm{mês}$ & 0 & 0 & 15.000 \\
\hline Condutividade & $\mu \mathrm{S}$ & 2.000 & $>30.000$ & $<20.000$ \\
\hline Temperatura & $\mathrm{oC}$ & $<45$ & 70 & 65 \\
\hline Lama & Caçamba/semana & 3 & 2 & 6 \\
\hline $\mathrm{kg}$ & - & 0,045 & 0,03 & 0,09 \\
\hline Lama/gusa & - & 4 & 10 & 3 \\
\hline Manta do filtro & telas/mês & $\mathbf{X}$ & $\mathbf{1 0} \mathbf{X}$ & $\mathbf{3 X}$ \\
\hline Custo & $\mathbf{R}$ \$mês & & &
\end{tabular}

Os itens mencionado no quadra são : a soda é utilizada para corrigir o pH que normalmente cai em função dos gases do forno. O polímero auxiliar de floculação e para reduzir a dosagem do PAC; o dispersante uso continuo para melhor evitar incrustação nos bicos. A condutividade e a temperatura controle do processo. A lama e manta dos filtros a vácuo são itens de controle no processo de retirada de lama do espessador. Na figura 06 e 07 podemos constatar a água de saída com aspecto muito bom com as condições da coluna atual. 


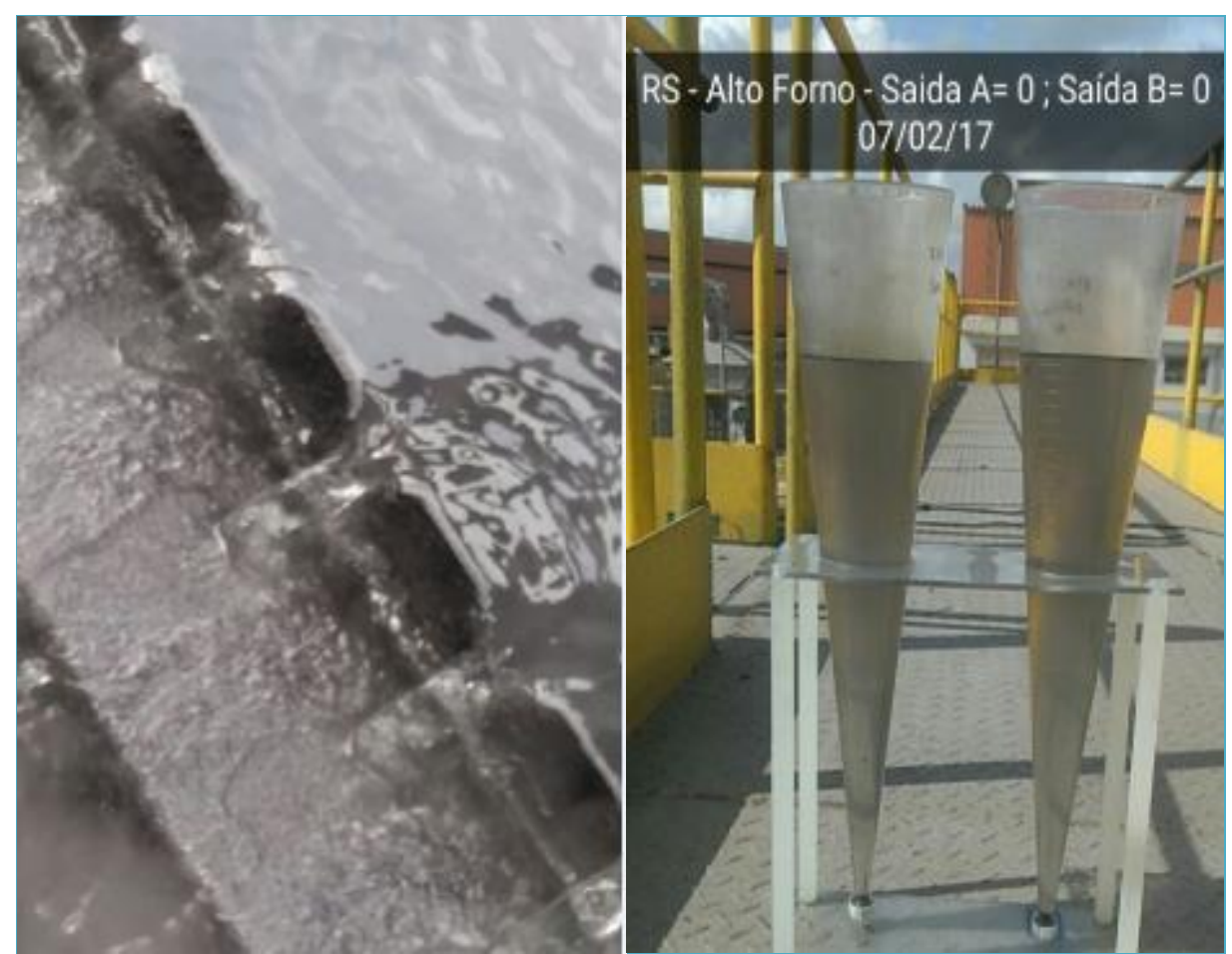

Figura 06 e 07 : Vista da água da saída do espessador e teste de RS igual a zero.

Controle na densidade da lama foi fundamental para garantir a operação do filtro funcionando 24 horas e com excelente retirada de lama (espessura da lama ficou entre 5 a $8 \mathrm{~mm}$ ). A espessura da lama permitiu uma maior remoção de sólidos no primeiro giro do filtro a vácuo. A maior evidência da remoção da lama é a amperagem nos espesssadores abaixo da faixa $(<3,5 \mathrm{~A}$ ), mostrado na figura 08 , o que confirma a eficiência da remoção de sólidos e garantindo uma campanha sem limpeza de espessador por mais de 8 meses. A economia gerada pela não contratação de equipamentos e empresa terceirizada para fazer o trabalho. A qualidade da lama formada também permite um menor desgaste da manta (aumento do tempo de uso de 2 dias para 10 dias)

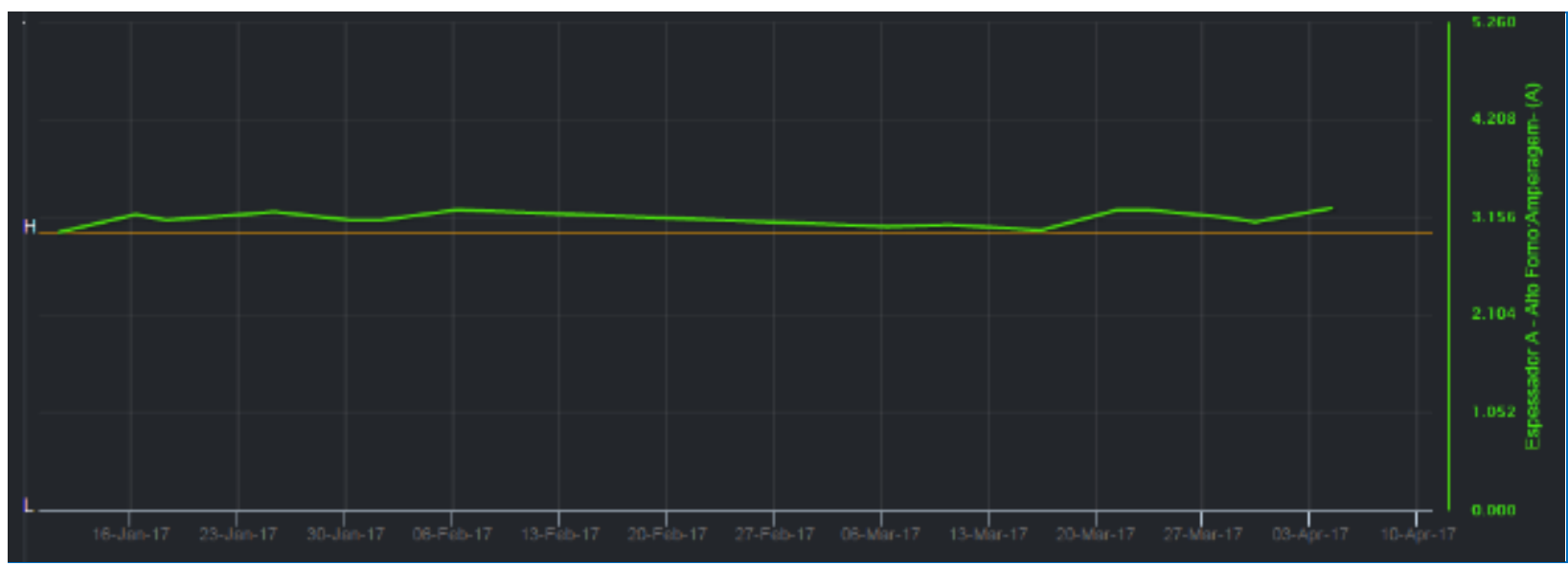

Figura 08 : Amperagem do espessador ao longo do ano de 2016 e $2017^{6}$. 


\section{CONCLUSÃO}

Concluímos que o trabalho em equipe foi excelente, proporcionando uma operação mais tranquila e com resultados excelentes para o processo. Proporcionou uma economia de água da ordem de $18.000 \mathrm{~m}^{3} / \mathrm{mês}$ (4X). O efluente dentro da especificação no tocante a qualidade dos parâmetros recomendado e uma economia global de $11 \mathrm{X} / \mathrm{mês}$. Os valores não foram mencionados em função de não poderem ser divulgados/partilhados ao público, por ser um tema confidencial e restrito a CSP. Resolvemos apenas mostrar a ordem de grandeza proporcional da redução de custo obtida.

Outra lição aprendida foi que nem sempre o que funciona bem em algum lugar do mundo, pode ser reproduzido de maneira idêntica em outra região. As particularidades de cada local devem ser estudadas e em muitas vezes exige adaptações e ajuste para encontrar a solução ideal.

\section{Agradecimentos}

Agradecimento a toda equipe da CSP de operação e manutenção pelo insistente trabalho de apoio e dedicação ao longo de 2016 e 2017 e do laboratório da CSP no tocante as análises de acompanhamento.

\section{REFERÊNCIAS}

1 Manual interno da Cia Siderúrgica do Pecem, Manual descritivo da operação, ano de 2015, capitulo 10 , página 7.

2 Material interno da CSP, Check list da unidade de lavagem de gases da CSP, ano de 2016,

3 Hancock, Bill, Thickener Operation, 26/01/2015,. Disponível em : http://www.zerodayllc.com/blog/2015/01/26/thickener-operation/

4 Material Interno da CSP, Escopo de apresentação de proposta de tratamento de água para Alto Forno, ano 2015, anexo 1, página 8.

5 Material interno da CSP, Relatório de análise do laboratório central da CSP, ano de 2016. Enviado eletronicamente para a área.

6 Insight GE, Software de controle estatístico do GCP, ano 2016 e 2017, disponível na plataforma WEB para CSP. 